\title{
Corrected model for transport in static diffusion chamber
}

M. Anisimov

S. Shandakov

V. Pinaev

A. Belyshev

Richard H. Heist

Fairfield University, rheist@fairfield.edu

Follow this and additional works at: https://digitalcommons.fairfield.edu/engineering-facultypubs Copyright 2000 American Institute of Physics

The final publisher PDF has been archived here with permission from the copyright holder.

\section{Repository Citation}

Anisimov, M.; Shandakov, S.; Pinaev, V.; Belyshev, A.; and Heist, Richard H., "Corrected model for transport in static diffusion chamber" (2000). Engineering Faculty Publications. 147.

https://digitalcommons.fairfield.edu/engineering-facultypubs/147

\section{Published Citation}

Anisimov, M., Shandakov, S., Pinaev, V., Belyshev, A., \& Heist, R. (2000, August). Corrected model for transport in static diffusion chamber. Nucleation and Atmospheric Aerosols 2000, AIP Conference Proceedings, Vol. 534(1), pp. 358-361. AIP. https://doi.org/10.1063/1.1361886.

This item has been accepted for inclusion in DigitalCommons@Fairfield by an authorized administrator of DigitalCommons@Fairfield. It is brought to you by DigitalCommons@Fairfield with permission from the rightsholder(s) and is protected by copyright and/or related rights. You are free to use this item in any way that is permitted by the copyright and related rights legislation that applies to your use. For other uses, you need to obtain permission from the rights-holder(s) directly, unless additional rights are indicated by a Creative Commons license in the record and/or on the work itself. For more information, please contact digitalcommons@fairfield.edu. 


\title{
Corrected Model for Transport in Static Diffusion Chamber
}

\author{
M. Anisimov ${ }^{1}$, S. Shandakov ${ }^{1}$, V. Pinaev ${ }^{2}$ A. Belyshev ${ }^{1}$, and R. Heist ${ }^{3}$ \\ 'Kemerovo State University, 6 Krasnaya Str., 650043 Kemerovo, Russia. \\ ${ }^{2}$ Kemerovo Institute of Commerce, 34 Kuznetskiy Prospect, 650099 Kemerovo, Russia \\ ${ }^{3}$ Nucleation Laboratory, Manhattan College, Riverdale, NY, USA
}

\begin{abstract}
Abastract. In present consideration for the static diffusion chamber the model of mass and heat transfer and its analytical solution are presented for the pseudo-open in one direction system.
\end{abstract}

Experimental research of homogeneous nucleation kinetics of supersaturated vapors is based on the determination of relation between nucleation rate and, as a rule, vapor supersaturation (activity) under the constant other parameters. The static diffusion chamber (SDC) allowed direct measurements of nucleation rates could be a powerful instrument for nucleation study. The vapor supersaturation is calculated by using the heat and mass transfer models. Recently the effect of the radial vapor flow to sidewall $^{1,2}$ has been calculated. A good agreement between one-dimensional and two-dimensional models (with zero mass average velocities at boundary) was received. It is customary to accept that the diffusion flux is zero at the chamber boundary where vapor is in equilibrium with wet wall. But SDC can be considered as an open system in one direction because of the mass transport of vapor from one boundary (hot plate) to other (cold plate). For this reason idea of a zero vapor flow to boundary is not correct. The model with zero vapor flow may be realized in the case of zero vapor concentration only. At nonzero vapor concentration one have for this model three boundary conditions (first and second are respectively equilibrium concentration values on hot and cold plates, third is zero value of the vapor diffusion flux) for one differential equation of 2-d order with two integration constants only. Recently the nonzero diffusion vapor flux to boundary was accounted in the initial transport equations 3 . Presented numerical solution ${ }^{3}$ is in a good agreement with an existing one - dimension model based on numerical solution of the Stefan-Maxwell and heat transfer equations. In present consideration the model of mass and heat transfer and its analytical solution are presented for the open in one direction system. Calculated values for vapor supersaturation are compared with results of Czech group by Rudek et al. ${ }^{4}$ on the example of n-pentanol nucleation rates.

According to Hirschfelder et al. ${ }^{5}$ system of mass - heat transfer equations in the case of axialsymmetry for the binary mixture stationary flow at the relatively small mass average velocities may be presented as

$$
\begin{array}{llc}
\frac{d(\rho u)}{d z}=0 & \frac{d p}{d z}=0 & \rho u \frac{d c}{d z}=-\frac{d J_{z}}{d z} \quad \rho u \frac{d h}{d z}=-\frac{d q_{z}}{d z}, \\
J_{z}=-\rho D_{12}\left(\frac{d c}{d z}+k_{T 2} \frac{M_{1} M_{2}}{M^{2}} \frac{d \ln T}{d z}\right), & q_{z}=-\lambda \frac{d T}{d z}+\left(h_{2}-h_{1}+k_{T 2} \frac{p}{(1-c) c \rho}\right) J_{z} .
\end{array}
$$

Here $p, \rho$ and $u$ are the total pressure, density and the average mass velocity, respectively; $c$ is the vapor mass fraction; $h, h_{1}$ and $h_{2}$ are specific enthalpies of mixture, gas and vapor, respectively; $J_{z}$ and $q_{z}$ are the axial mass flux of vapor and heat flux, respectively; $T$ is temperature; $\lambda, D_{I 2}$ and $k_{T 2}$ are heat conductivity, binary diffusion coefficient of mixture and thermal diffusion factor of vapor, respectively; $M_{1}, M_{2}$ and $M=\left[(I-c) / M_{1}+c / M_{2}\right]^{-1}$ are molar weights of gas, vapor and mixture, respectively.

It is assumed that at given temperatures on hot plate, $T_{b}$, and cold plate, $T_{l}$, the vapor concentrations, as boundary conditions, are equal to the equilibrium vapor concentrations, $c_{0}$ and $c_{1}$, respectively. The boundary average mass velocity is determined from condition of not penetrating gas through boundaries. At $z=0$ this condition is

$$
\left(1-c_{0}\right) \rho_{0} u_{0}-J_{z 0}=0 \text {. }
$$

CP534, Nucleation and Atmospheric Aerosols 2000: $15^{\text {th }}$ Int.' $l$ Conf., edited by B. N. Hale and M. Kulmala (C) 2000 American Institute of Physics 1-56396-958-0/00/\$17.00 
Accepted that specific enthalpy $h=(1-c) h_{1}+c h_{2}$. Equation (1) after integration with the taken into account the boundary condition (3) may be expressed as

$$
\begin{gathered}
\rho u=\rho_{0} u_{0}=\frac{J_{z 0}}{1-c}, \quad p=p_{0}, \\
\frac{J_{z 0}}{1-c_{0}}\left(c-c_{0}\right)=-\left(J_{z}-J_{z 0}\right), \\
\frac{J_{20}}{1-c_{0}}\left(h_{1}-h_{10}+c\left(h_{2}-h_{1}\right)-c_{0}\left(h_{20}-h_{10}\right)\right)=-\left(q_{z}-q_{20}\right) .
\end{gathered}
$$

Here and further values at reference plate (at $z=0$ ) are indicated by index "zero". After transformation the equation (5) can be written as

$$
J_{z}=\frac{1-c}{1-c_{0}} J_{20}
$$

Substituting (7) into (2) we have

$$
\begin{aligned}
& \frac{d c}{d z}=-\frac{1-c}{1-c_{0}} \frac{J_{z 0}}{\rho D_{12}}\left(1+\frac{k_{T 2}}{T} \frac{M_{1} M_{2}}{M^{2}} \frac{d T}{d c}\right)^{-1}, \\
& q_{z}=-\lambda \frac{d T}{d z}+\left(h_{2}-h_{1}+k_{T 2} \frac{p_{0}}{(1-c) c \rho}\right) \frac{1-c}{1-c_{0}} J_{z 0} .
\end{aligned}
$$

After substitution of heat fluxes, $q_{z}$ and $q_{z 0}$, in the form (9) into (6) and after the result transformation we receive the next equation:

$$
\lambda \frac{d T}{d z}-\lambda_{0}\left(\frac{d T}{d z}\right)_{0}+\left(h_{20}-h_{2}+k_{T 2}^{0} \frac{p_{0}}{c_{0} \rho_{0}}-k_{T 2} \frac{p_{0}}{c \rho}\right) \frac{J_{z 0}}{1-c_{0}}=0 .
$$

Specific heat capacity, $c_{p 2}$, of vapor at constant pressure and temperature is constant. Using state equation for mixture, $P / \rho=R T / M$, equation (10) can be rewritten to form

$$
\lambda \frac{d T}{d c} \frac{d c}{d z}-\lambda_{0}\left(\frac{d T}{d c}\right)_{0}\left(\frac{d c}{d z}\right)_{0}=\frac{J_{z 0}}{1-c_{0}} c_{p 2}\left(T-T_{0}+\frac{\gamma_{2}-1}{\gamma_{2}}\left(\frac{k_{T 2} M_{2} T}{c M}-\frac{k_{T 2}^{0} M_{2} T_{0}}{c_{0} M_{0}}\right)\right) .
$$

Here gas constant is presented through adiabatic ratio for the vapor as $R=c_{p 2}\left(\gamma_{2}-1\right) / \gamma_{2}$.

One can substitute expressions (8) for $d c / d z$ and $(d c / d z)_{0}$ into equation (11). After transforming we come to

$$
\begin{gathered}
J_{z 0} \cdot\left[\frac{L e(1-c)}{K+d c / d T}-\frac{L e_{0}\left(1-c_{0}\right)}{K_{0}+(d c / d T)_{0}}-\left(T-T_{0}+\frac{\gamma_{2}-1}{\gamma_{2}}\left(\frac{k_{T 2} M_{2} T}{c M}-\frac{k_{T 2}^{0} M_{2} T_{0}}{c_{0} M_{0}}\right)\right)\right]=0 \\
K_{0}=\frac{k_{\Gamma 2}^{0} M_{1} M_{2}}{M_{0}^{2}}, \quad K=\frac{k_{T 2} M_{1} M_{2}}{M_{0}},
\end{gathered}
$$

where Lewis numbers, respectively $L e$ and $L e_{0}$, are expressed as

$$
L e=\frac{\lambda}{\rho D_{12} c_{12}}, \quad L e_{0}=\frac{\lambda_{0}}{\rho_{0} D_{12}^{0} c_{p 2}} .
$$

The equation (12) has two solutions, namely, the first, for zero diffusion flux, $J_{z 0}$, and the second, for nonzero vapor flux, $J_{z 0}$. One can get the dimensionless temperature, $t=T / T_{0}$, and designations

$$
A=\left(\frac{d c}{d t}\right)_{0}+K_{0}, \quad B(\tau, c)=\frac{1}{L e_{0}\left(1-c_{0}\right)}\left[1-\tau+\frac{\gamma_{2}-1}{\gamma_{2}} \frac{M_{2}}{M_{0}}\left(\frac{k_{T 2}^{0}}{c_{0}}-\frac{M_{0}}{M} \frac{\tau \cdot k_{T 2}}{c}\right)\right],
$$

we rewrite equation (12) for the case of nonzero diffusion flux to form 


$$
\frac{d c}{d t}=\frac{A}{1+A \cdot B(\tau, c)} \frac{1-c}{1-c_{0}} \frac{L e}{L e_{0}}-K .
$$

Thus we received a first order differential equation, where integrating constant $A$ is defined from one of two boundary conditions for vapor concentration at plates with different temperatures, $T_{0}$ and $T_{1} . L e, K$ and $B(\tau, c)$ are not simple functions of vapor concentration and temperature in common case.

Usually experiments in the static diffusion chamber are doing in atmosphere of light gas (helium). In this case the gas molar weight is much smaller than vapor one $\left(M_{1}<<M_{2}\right)$ and vapor mass fraction is not closely to unit. In the frame of presentation by Hirschfelder et al..$^{5}$ work the heat conductivity of mixture in the form of Vassiljev's equation ${ }^{6}$ may be presented as

$$
\lambda=\lambda_{1}(1-c) \frac{M}{M_{1}} .
$$

In the same approximation, with account the equation (15), the Lewis numbers can be expressed through interaction integrals, $\Omega,{ }^{5}$ as

$$
\begin{gathered}
\frac{L e}{L e_{0}}=\frac{1-c}{1-c_{0}}, \\
L e_{0}=\frac{25}{8} \cdot \frac{\gamma_{2}-1}{\gamma_{2}}\left(\frac{4}{15} \frac{c_{V 1} M_{1}}{R}+\frac{3}{5}\right) \sqrt{\frac{2 M_{2}}{M_{1}+M_{2}}} \cdot \frac{M_{2}}{M_{1}}\left(\frac{\sigma_{12}}{\sigma_{1}}\right)^{2} \frac{\Omega^{(1,1)}\left(k T_{0} / \varepsilon_{12}\right)}{\Omega^{(2,2)}\left(k T_{0} / \varepsilon_{1}\right)},
\end{gathered}
$$

where $c_{V 1}$ is a specific heat capacity of gas at constant volume; $s$ and $e / k r$ are force constants for the Lennard-Jones (6-12) potential. According Hirschfelder et al. ${ }^{5}$, thermal diffusion factor of vapor can be present as

$$
k_{\mathrm{T} 2}=c(1-c) f \frac{M^{2}}{M_{1} M_{2}}, f \approx \frac{6 \gamma_{2} L e_{0}}{5\left(\gamma_{2}-1\right)} \frac{M_{1}}{M_{2}}\left(c_{12}^{*}-5 / 6\right),
$$

where $\quad c_{12}^{*}$ is function of $T_{12}=k T / e_{12}$, expressed through the interaction integrals, $\Omega$.

For $\mathrm{n}$-pentanol-helium system in present work is used extrapolation of thermal diffusion factor as in work $^{4}$

$$
f^{\prime}=\left(\left(-0.7272-\frac{T}{16.36-0.2882 \cdot T}\right) \cdot\left(1.12281-c \frac{M}{M_{2}}\right)+0.089303\right)^{-1}
$$

With account of equations (16) and (17) the equation (14) may be represented as

$$
\begin{gathered}
\frac{d}{d \tau}\left(\frac{1-c_{0}}{1-c}\right)=\frac{A_{0}}{1+A_{0} B_{0}}-c \cdot \frac{f}{\tau} \cdot \frac{1-c_{0}}{1-c}, \\
A_{0}=\frac{(d c / d t)_{0}+c_{0} f}{1-c_{0}}, \quad B_{0}=\frac{1-\tau}{L e_{0}}\left[1+f \frac{\gamma_{2}-1}{\gamma_{2}} \frac{M_{0}}{M_{1}} \frac{1-c_{0}}{1-\tau}\left(1-\tau \cdot \frac{M}{M_{0}} \frac{1-c}{1-c_{0}}\right)\right] .
\end{gathered}
$$

Solution for equation (18) is

$$
\begin{aligned}
& c^{(1)}=1-\left(1-c_{0}\right)\left(1-L e_{0} \ln \left(1+\frac{1-\tau}{1-\tau_{1}}\left(\exp \left(\frac{c_{0}-c_{1}}{1-c_{1}} L e_{0}^{-1}\right)-1\right)\right)\right)^{-1}, \\
& y^{(n)}=\left(G^{(n)}\left(1, \tau_{1}\right)-\frac{1-c_{0}}{1-c_{1}}\right) \frac{F^{(n)}(1, \tau)}{F^{(n)}\left(1, \tau_{1}\right)}-G^{(n)}(1, \tau)+1, \quad c^{(r)}=1-\frac{1-c_{0}}{y^{(n)}}, \\
& F^{(n)}(1, \tau)=\int_{1}^{\tau}\left(1+A_{0}^{(n-1)} B_{0}^{(n)}\right)^{-1} d \tau, \quad G^{(n)}(1, \tau)=\int_{1}^{\tau} c^{(n-1)} \frac{f}{\tau} \frac{1-c_{0}}{1-c^{(n-1)}} d \tau, \\
& A_{0}^{(1)}=\frac{L e_{0}}{1-\tau_{1}}\left(\exp \left(\frac{c_{0}-c_{1}}{1-c_{1}} L e_{0}^{-1}\right)-1\right), \quad A_{0}^{(n)}=\left(G^{(n)}\left(1, \tau_{1}\right)-\frac{1-c_{0}}{1-c_{1}}\right) / F^{(n)}(1, \tau),
\end{aligned}
$$




$$
B_{0}^{(n)}=\frac{1-\tau}{L e_{0}}\left[1+f \frac{\gamma_{2}-1}{\gamma_{2}} \frac{M_{0}}{M_{1}} \frac{1-c_{0}}{1-\tau}\left(1-\tau \cdot \frac{M^{(n-1)}}{M_{0}} \frac{1-c^{(n-1)}}{1-c_{0}}\right)\right], n=23, \ldots
$$

The solution (20) of equation (18) is for zero thermal diffusion factor $(\mathrm{f}=0$ ). The solution (21) gives the $n$-order iteration ones $(n=2,3,4 \ldots)$ with account of thermal diffusion effect. The present calculations were performed for 4 th order iteration $(n=4)$.

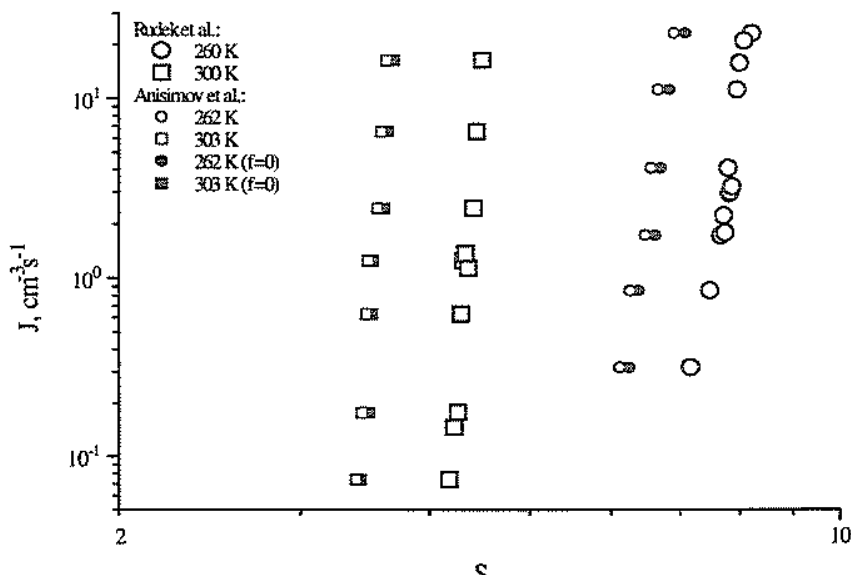

Fig. 1. Nucleation rates, J, versus vapor supersaturation, S, (Rudek et al.) and the same data calculated with the present research assumptions.

To evaluate difference between two treatments, namely with account of the interplate vapor flux and without it, the calculations of vapor supersaturations based on the experimental $n$-pentanol nucleation results were done. Present calculations was done at the same boundary conditions as by Rudek et al. ${ }^{4}$ The value of supersaturation was corresponded the maximum of theoretical nucleation rate calculated by using the classical nucleation theory:

$$
J_{\text {theor }}=\frac{V}{(k T)^{2}} \sqrt{\frac{2 \sigma}{\pi \cdot m}}: P^{2} \cdot \exp \left(-16 \pi \cdot \sigma^{3} \cdot V^{2} / 3(k T)^{3} \cdot(\ln S)^{2}\right),
$$

where $\sigma$ is the surface tension; $m, V$ are molecular mass and volume, and $S$ is the vapor supersaturation ratio (or vapor activity), $P$ is the partial vapor pressure, $T$ is the nucleation temperature, $k$ is the Boltzmann's constant. As seen in fig. 1 this difference is very large even for relatively small mass vapor fraction at temperature $260 \mathrm{~K}$. Herewith the calculated nucleation temperatures are larger as ones in Rudek et al. ${ }^{4}$ work. Our calculations are done with and without the thermal diffusion influence. The thermal diffusion influence on transport process is not too essential.

\section{REFERENCES}

I. Bertelsmann, A. and Heist, R.H. J.Chem. Phys., 106, 610; (1997).

2. F. T. Ferguson and J.A. Nuth, J. Phys. Chem. 111, 8013 (1999).

3. Stratmann, F., Zdimal, V., Wilck, M., and Smolik, J. J.Aerosol Sci., 30, Suppl.1, S75-S76. (1999)

4. Rudek, M., Katz, J.L., Vidensky, I.Y., Zdimal, V., and Smolik, J. $I$ Chem Phys. 111(8), 3623. (1999)

5. Hirschfelder, J.O., Curtiss, C.F., and Bird, R.B. Molecular Theory of Gases and Liquids. Wiley, NY. (1954)

6. Reid R.C., Prausnitz J.M., and Sherwood T.K. The properties of gases and liquids. $3^{\text {td }}$ ed. McGrow-Hill, New York, p. 508 (1977) 